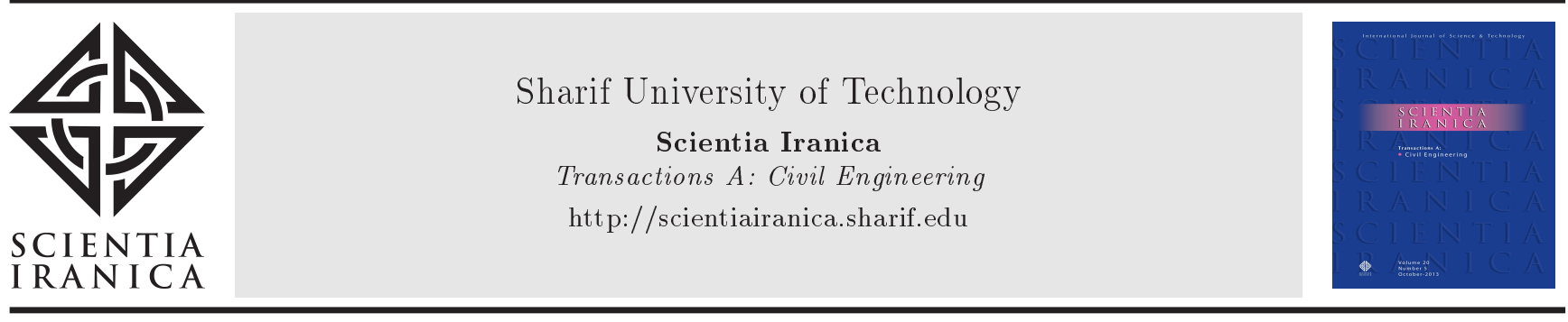

\title{
Developing a new algorithm (G-JPSO) for optimal control of pumps in water distribution networks
}

\author{
R. Rajabpour ${ }^{\mathrm{a}}$, N. Talebbeydokhti ${ }^{\mathrm{b}, *}$, and Gh.R. Rakhshandehroo ${ }^{\mathrm{c}}$ \\ a. Department of Civil Engineering, Science and Research Branch, Islamic Azad University, Tehran, Iran. \\ b. Department of Civil and Environmental Engineering, Environmental Research and Sustainable Development Center, Shiraz \\ University, Shiraz, Iran. \\ c. Department of Civil and Environmental Engineering, Shiraz University, Shiraz, Iran.
}

Received 28 May 2017; received in revised form 12 December 2017; accepted 2 July 2018

\section{KEYWORDS \\ Optimization; Pumping station; Operation; \\ Water distribution networks; \\ G-JPSO.}

\begin{abstract}
Meta-heuristic methods have been widely used for solving complex problems, recently. Among these methods, Jumping Particle Swarm Optimization (JPSO) is regarded as a promising algorithm. However, in order to achieve more robust performance, the probability of solving the graph-based problems is modified by changing the jumping nature of this algorithm. A new algorithm called G-JPSO is presented, which is evaluated by solving Fletcher-Powell function and optimal control of pumps in water distribution network problems. In addition to the reduction of electricity cost and the problem limitations such as minimum required pressure in each node, minimum and maximum height of tanks should also be considered. Moreover, another limitation on the objective function was performed that includes the maximum times of turning the pumps on and off. In order to determine the pumps' optimal operation, an optimization-simulation model based on the optimization algorithms G-JPSO and JPSO is developed. This proposed model is used for determining an optimal operation program of Van Zyl distribution network. The comparison carried out between the results of our proposed algorithm and those of the similar algorithms, including ant colony, genetic, and JPSO, shows the high ability of the presented algorithm in finding near-optimum solutions with reasonable computation costs.
\end{abstract}

(C) 2020 Sharif University of Technology. All rights reserved.

\section{Introduction}

Optimization in mathematics and computer science is a process of selecting or finding the best member in a set of available options. Each process has optimizing potential, and complicated issues can be modeled in engineering, economic, and commercial sciences such as optimizing issues. The objective of modeling such issues is to minimize the time, cost, and risk or to maximize the benefit, quality, and efficiency. In

*. Corresponding author.

E-mail address: taleb@shirazu.ac.ir (N. Talebbeydokhti)

doi: $10.24200 /$ sci. 2018.20703 recent years, the researchers have found proper findings in most complicated optimization issues by applying innovative ways.

The related algorithms are classified into two classes of definite and probable forms [1]. Definite algorithms are local searching methods based on a gradient, requiring basic movement information for finding possible solutions [2]. In such issues in which solution space is non-convex, it is not simple to find a global optimal solution using these algorithms. In other words, some non-convex problems include multiple local optimal solutions in their solving space. In such problems, the quality of final solutions needs definite initial values. These algorithms are inspired by natural phenomena and do not need moving information for 
finding any solution. The most important probable optimal algorithms are genetic algorithm, forbidden search, group ingredients optimization, and ant colony simulation. In recent years, hybrid global-local algorithms were applied successfully for solving non-convex problems [2]. These algorithms combine the local and global searching aspects in their structures for efficient searching. In these algorithms, the searching process starts with multiple points, and searching for solution space will be carried out with these points; then, local searching algorithms find an optimal solution in these spaces. The searching space could be in the continuous or discrete form. One of these algorithms, which is very useful in water engineering domain, is Jumping Particle Swarm Optimization (JPSO). The first aim of this paper is to develop the aforementioned algorithm in discrete space in order to solve discrete graphic problems in it and, then, measure its performance in pumps optimal operation problems in water distribution networks.

The main portion of the energy consumed in water distribution networks is related to the available pumps in those networks. By determining a proper schedule for operation of the pumps in the water transferring system, the energy cost can be reduced by a significant amount.

Since the constraints and objective functions are non-linear and the number of decision variables and constraints is simply high, the problem of pumps optimal operation in a water distribution network is a part of large non-linear problems. The objective function is to minimize the operational objectives from pumping stations during a planning horizon, in which the system pumps out water during this time [3].

Recently, many studies have been conducted on pumps optimal performance and pumping stations. For example, Mackle et al. (1995) performed some researches on the optimal operation of pumping stations (consumed electricity cost). In their research, genetic algorithm was used as an optimization tool [4]. Using genetic algorithm, Rodin and Moradi-Jalal (2002) optimized pumping stations for minimizing the cost during a day [5]. Moradi-Jalal (2003) also presented a new model for designing optimal operation from agricultural pumping stations by utilizing genetic algorithm. In their model, by considering leveraging characteristics of pumps and curve in the downstream, different combinations of pumps are made that can meet the needs; then, the one with the least cost will be selected as the final option among the choices [6]. Using PSO algorithm, Rajabpour and Afshar presented an optimal operation program from continuous pumping stations with variable speed pumps to minimize energy cost [7]. Van Zyl et al. (2004) conducted an optimal operation of distribution network pumps by using genetic algorithm [8]. López-Ibáñez (2008) optimized the operation of pumps by considering the times of turning on and off as a limitation using ant colony algorithm [9]. Bozorg Hadad and Marino (2007) used dynamic and static penalty functions for Honey-Bee Mating Optimization (HBMO) algorithm [10]. Sanda-Carmen et al. (2010) determined the optimal operation instruction of a loop network with variable demands and variable speed pumps using ant colony optimization algorithm [11]. Combining linear programing with genetic algorithm, Rasoulzadeh Gharibdosti et al. optimized pumping stations for optimal operation. In comparison with ant colony algorithm and genetic algorithm, their combined algorithm showed better results [12].

Hashemi et al. (2011) used variable speed pumps in distribution networks in order to decrease energy consumption cost. Their studies about Verdaver distribution network resulted in a $5.43 \%$ decrease in energy consumption cost [13]. Following this, Hashemi et al. (2012) showed that the use of variable speed pumps in distribution networks, in addition to the reduction of energy cost, increased the reliability of water-supplying system [14]. In order to study a decrease in leakage and pressure in water distribution networks, Mehzad et al. (2012) determined the optimal operation program for pumping stations. Their results showed that a reduction in energy cost, leakage, available pressure can be achieved through smart programming [15]. Hashemi et al. (2014) used the ant colony algorithm to optimize pumping program in water distribution network using variable speed pumps in the status of water demanding changes during a day. In addition to the methods proposed for reduction in searching space, it was shown that the use of variable speed pumps could lead to greater saving (about 10\%) in pumping energy cost, compared with fixed-speed pumps [16]. In addition, concerning pumps operation schedule in a water distribution network, the studies of Abdelmeguid and Ulanicki (2010), Skworcow et al. (2010), Fiorelli et al. (2012), and Paluszczyszyn et al. (2013) can be considered [17-20].

Applying innovative changes in jumping nature of JPSO algorithm, this paper adds the ability to solve discrete graph problems. Then, to investigate the capability of the proposed algorithm, the mathematical complex function of Fletcher-Powell (five dimensions) and the pumps' optimal control issue in water distribution networks will be studied, and the results will be compared with those of ant colony and genetic algorithm.

\section{A review on non-continuous PSO algorithms}

For the first time, by proposing a simple change in standard PSO nature, Kennedy and Eberhart (1997) used this algorithm for solving non-continuous problems [21]. In standard PSO, speed, in this case, means 
particle movement vector in searching space; however, in discrete space, this meaning does not hold anymore. In this developed algorithm, the threshold speed is a possibility based on which variable $X_{i j}$ has the value of 0 or 1 .

In this algorithm, updating velocity is mostly similar to the standard PSO; however, the weight inertia parameter $(w)$ is not applied to it as follows:

$$
v_{i j}^{\prime}=v_{i j}+c_{1} \operatorname{rand}()\left(b_{i j}-x_{i j}\right)+c_{2} \operatorname{rand}()\left(g_{j}^{*}-x_{i j}\right) .
$$

In order to specify a new value for $X_{i j}$, at first, the speed value should be changed to a number between zero and one. For this purpose, sigmoid function $S(X)$ will be used in the following:

$$
S(x)=\frac{1}{1+e^{x}} .
$$

Then, the value of variable $X_{i j}$ will be selected accidentally through Eq. (3):

$$
x_{i j}^{\prime}= \begin{cases}1 & \text { if }\left(\operatorname{rand}()<S\left(v_{i j}^{\prime}\right)\right) \\ 0, & \text { otherwise }\end{cases}
$$

Yang et al. (2004) and Al-Kazemi and Mohan (2002), based on PSO non-continuous algorithm of Kennedy and Eberhart, presented similar PSO algorithms for solving problems coded as 0 and 1 . The main difference between these two algorithms and non-continuous PSO algorithm of Kennedy and Eberhart lies in 'how the speed is updated' $[22,23]$.

Morono-Perez et al. (2007) innovated a new method based on PSO for solving combined optimal problems [24]. Since the speed vector cannot be described as a particle movement vector in a discrete space, it is simply disregarded in their method. In this method, a particle movement in a discrete space is described as a particle jumping from a response to another one. This jump can resemble the jump of a frog on lilies. The name of this algorithm is Jumping PSO (JPSO).

\subsection{Jumping PSO (JPSO)}

In this method, the position of a particle $X_{i}=$ $\left(X_{i 1}, X_{i 2}, \ldots, X_{i s}\right)$ ( $s$ is the number of decision variables) is studied as an acceptable response for the combined optimal problem. The particle position in each iteration will change through jumping from one response to another. There can be four kinds of jumping in any iteration, one of which will be selected. The first is an accidentally chosen jumping. The second is a jump toward the best previous position of particle $B_{i}=\left(b_{i 1}, b_{i 2}, \ldots, b_{i s}\right)$; the third is a jump toward the best position in the particle neighborhood $G_{i}=$ $\left(G_{i 1}, G_{i 2}, \ldots, G_{i s}\right)$, and the fourth is a jump toward the best position in iteration $G^{*}=\left(g_{1}, g_{2}, \ldots, g_{s}\right)$. In this method, $B_{i}, G_{i}$, and $G^{*}$ are called attractors. Components' position timing is described by Eq. (4):

$$
X_{i}=c_{1} X_{i} \oplus c_{2} B_{i} \oplus c_{3} G_{i} \oplus c_{4} G^{*} .
$$

This equation shows that, with the possibility of $c_{1}$, the jumping will be done accidentally (jumping type 1 ); with the possibility of $c_{2}$, the jumping will be formed toward $B_{i}$ (jumping type 2); with the possibility of $c_{3}$, the jumping will be formed toward $G_{i}$ (jumping type 3 ); with the possibility of $c_{4}$, the jumping will be formed toward $G^{*}$ (jumping type 4 ). The sum of values $c_{1}, c_{2}, c_{3}$, and $c_{4}$ is equal to 1 ; in each phase, however, only one of them will act. In each phase, the jumping will be done step by step. Suppose that one of the jumping kinds is selected for the considered particle with the selection possibility of $c_{i}$ and, in the first jumping step, one of the decision variables is selected accidentally with a steady possibility and its value, which will be changed. In jumping type 1 , the changing value of the decision variable is chosen accidentlly, while it is substituted with its same value in attractor in jumping types 2, 3, and 4 . Following the completion of the first step, the random number $\zeta$ with a steady possibility is selected between zero and one. If $\zeta$ is smaller than $c_{i}$, then another decision variable is selected accidentlly with a steady possibility and its value, which will be changed; otherwise, the jumping stops.

\section{The mechanism of the proposed algorithm}

The tree growing algorithm for solving the problem of optimal order of the pressurized irrigation network was first examined by Sami Kashkouli and Monem by applying innovative changes in the jumping nature of JPSO algorithm examined [25]. In this paper, by modeling based on the mentioned method and applying some additional changes, the ability to solve discrete graphic issues has been created. This algorithm is named G-JSPO, and its flowchart is presented in Figure 1. The changes applied to JPSO algorithm are of jumping nature, which will be described in detail in G-JSPO algorithm, as in the following.

In the phase of jumping in the $i$ th particle, at first, the particle (Figure 2(a)) and the attractor (a random subgroup from the total set of decision options) (Figure 2(b)) are prescribed, and a basic order is gained (a suborder from a maximum order) (Figure 2(c)). Then, a new response graph is selected from this basic order (Figure 2(d)). It should be mentioned that each created particle and, also, each created attractor represent a response graph. Figure 2 shows all the jumping steps toward the attractor described in Figure 1.

Since G-JPSO algorithm is proposed in order to empower JPSO to deal with graphic-based problems (such as the problems soluble using ACO algorithm) and to obtain better answers, a basic change is applied 


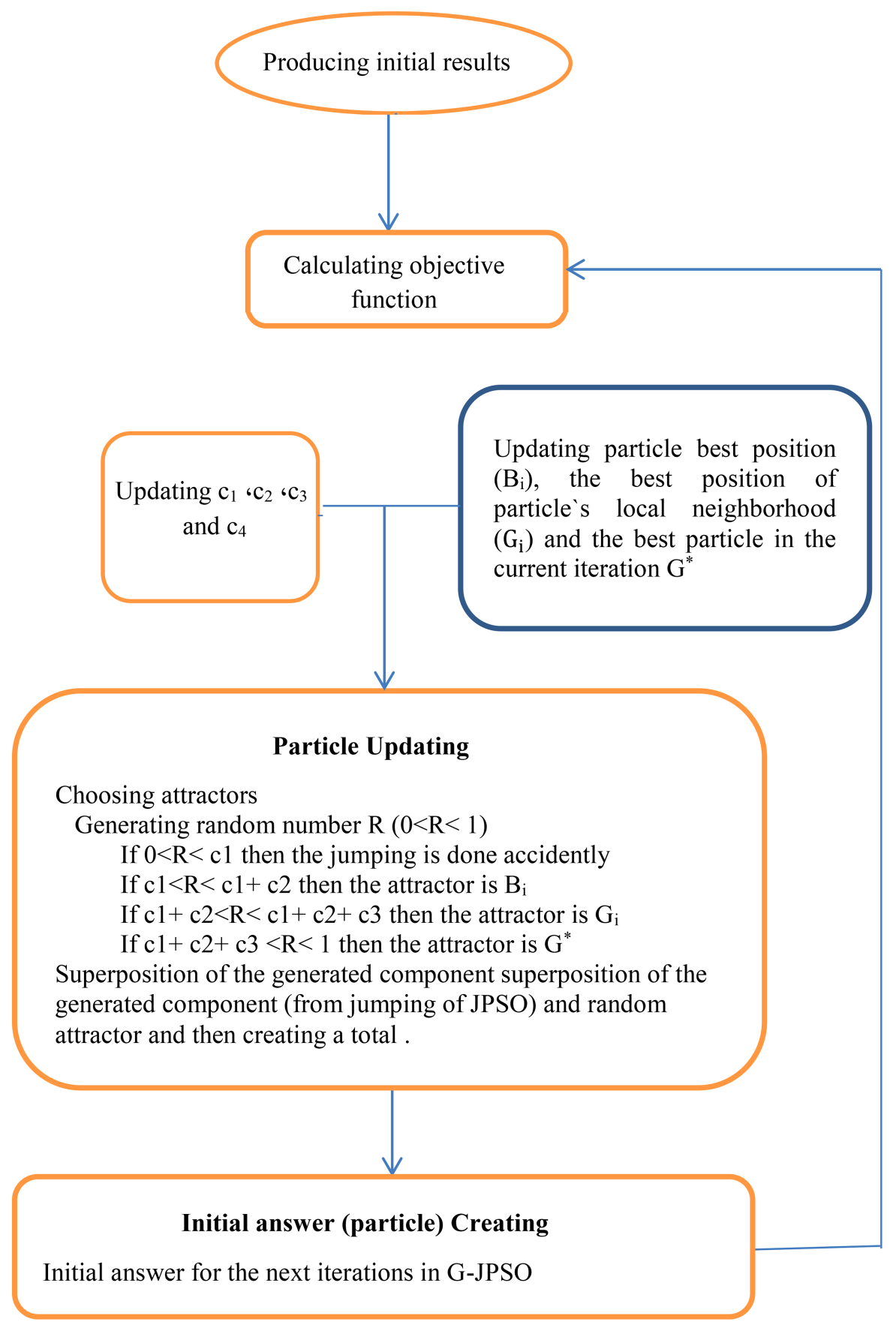

Figure 1. G-JPSO algorithm.

to the jumping section when the graph of the new answer is generated (compared to JPSO algorithm). Hence, in the components synchronization section of Figure 1, some explanations are added to illustrate this point. This section concerns (a) the superposition of the generated component (from jumping of JPSO) and a random attractor, (b) the creation of a total graph, and (c) the production of a new particle answer from the total graph (an initial answer for the next iterations in G-JPSO and calculating the objective function).
Figure 3 depicts a particle in G-JPSO algorithm. As is shown, in each decision point, the algorithm can select one of the numbers between 1 and 24 (daytime hours). It starts from the first decision point with on status and continues to the end. In each decision point, the sum of the chosen numbers should be less than 24 (total daytime hours is 24). For example, the selection particle in Figure 3 shows that the pump is on in the first hour; then, it is off for two hours; it is on for twelve hours and, then, it is off for one hour. One hour later, it 
(a) Particle

\begin{tabular}{|l|l|l|l|l|l|}
\hline 1 & 2 & 12 & 1 & 1 & 7 \\
+
\end{tabular}

(b) Attractor

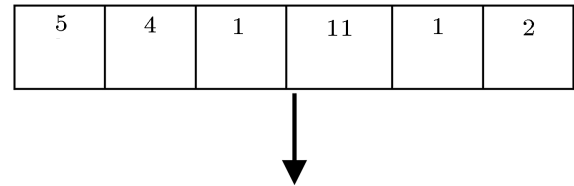

(c)

\begin{tabular}{|c|c|c|c|c|c|}
\hline 1 & 2 & 12 & 1 & 1 & 7 \\
\hline 5 & 4 & 1 & 11 & 1 & 2 \\
\hline
\end{tabular}

G-JPSO algorithm

(d) Options decision in G-JPSO

\begin{tabular}{|l|l|l|l|l|l|}
\hline 5 & 2 & 1 & 11 & 1 & 4 \\
\hline
\end{tabular}

Figure 2. Jumping and updating particle in G-JPSO.

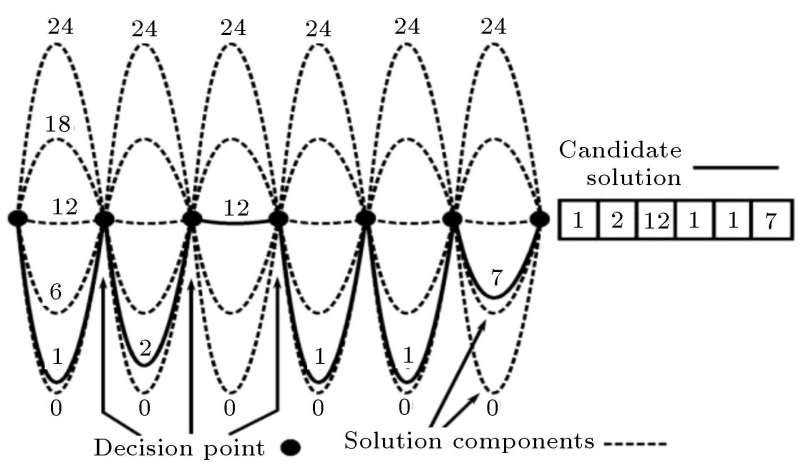

Figure 3. A particle in G-JPSO.

will be on and, finally, it is off for seven hours. It means that this pump is on and off for 3 times in daytime.

\section{Sample issues}

In order to study the ability of the proposed algorithm, at first, the complicated mathematical Fletcher-Powell function (5 dimensions) and, then, pumps optimal control issue in water distribution networks are investigated as the real optimizing problem.

\subsection{Minimizing the Fletcher-Powell function}

Fletcher-Powell function is a non-linear function and is nonpolar and inseparable from its variables [26]. In addition, an irregular distribution of local optimal points is another characteristic of this function. The form of this function is as follows:

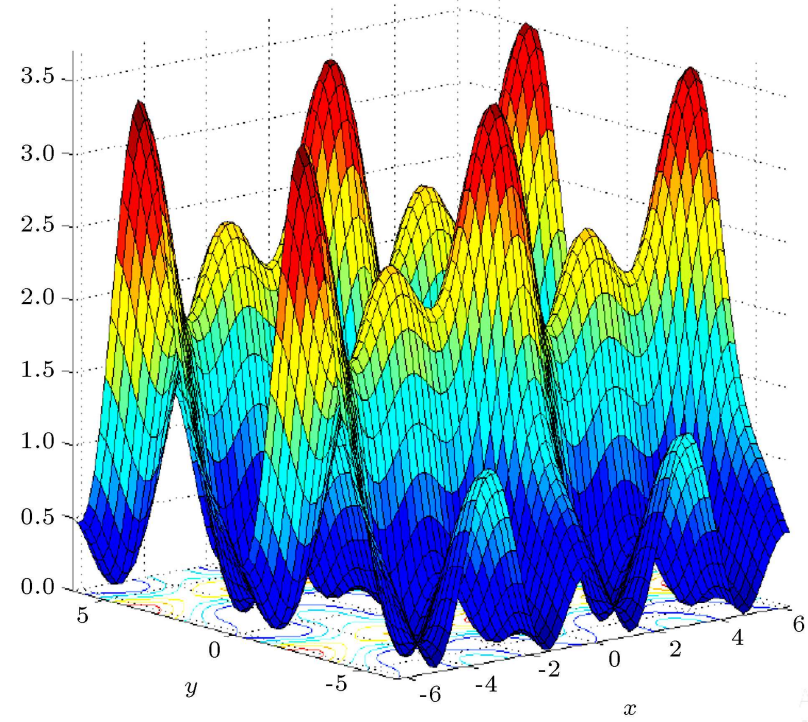

Figure 4. The two-dimensional Fletcher-Powell function.

$$
\begin{aligned}
& f(x)=\sum_{i=1}^{n}\left(A_{i}-B_{i}\right)^{2}, \\
& A_{i}=\sum_{j=1}^{n}\left(a_{i j} \sin \propto_{j}+b_{i j} \cos \propto_{j}\right), \\
& B_{i}=\sum_{j=1}^{n}\left(a_{i j} \sin x_{j}+b_{i j} \cos x_{j}\right), \\
& -\pi \leq x_{j} \leq \pi \quad(i=1,2, \ldots, n),
\end{aligned}
$$

where $a_{i j}$ and $b_{i j}$ are the random integers in the allowed range $[-100,100]$, and $\alpha_{i}$ is a random value in range $[-\pi, \pi]$. As is obvious at the first look, the total optimal value of this function is equal to 0 ; therefore, the decision variables will be attained as $x_{j}=\alpha_{j}(j=1,2,3, \ldots, n)$. However, owing to the complexities involved, nonpolar and inseparable variables, and the irregularity of a local optimum, its solution is difficult whether in a definite form or in a searching form. Figure 4 shows the mentioned (twodimensional) function $(n=2)$.

For modeling this problem with G-JPSO algorithm, the domain is divided into 11 equal classes. Statistical results of 15 different performances are shown in Table 1 by using G-JPSO algorithm.

Table 1. Statistical analysis of Fletcher-Powell function.

\begin{tabular}{lcc}
\hline \multicolumn{1}{c}{ Algorithm } & ACO* $^{*}$ & G-JPSO \\
\hline Middle answer & 3227.41 & $\mathbf{2 1 9 . 3 6 5 7}$ \\
The best answer & 723.74 & $\mathbf{5 0 . 3 2 7 1}$ \\
The worst answer & 7625.42 & $\mathbf{3 4 1 . 6 0 4 8}$ \\
\hline
\end{tabular}

* Jalali and Afshar (2005) [27]. 
Table 2. G-JPSO parameters.

\begin{tabular}{cccccc}
\hline $\begin{array}{c}\text { The number } \\
\text { of repetitions }\end{array}$ & $\begin{array}{c}\text { The number } \\
\text { of particles }\end{array}$ & C1 & C2 & C3 & C4 \\
\hline 1000 & 20 & 0.55 & 0.15 & 0.15 & $\mathbf{0 . 1 5}$ \\
\hline
\end{tabular}

In Table 2, the parameters of G-JPSO algorithm used in solving this mathematical function are listed.

Tables 1 and 2 demonstrate that, by assigning the proposed mechanism, the results are much better than those of ant colony algorithm.

\subsection{Pumps optimal control in distribution networks}

For determining pumps optimal operation program, a simulation-optimization model is proposed based on optimizing algorithm G-JPSO. In this model, the proposed algorithm was assimilated in Matlab area with the hydraulic part of EPANET model. A proper operation schedule includes the determination of on and off statuses of each pump in the considered time range. In addition to decreasing the electricity consumption cost, the need for consuming nodes and limitations such as minimal required pressure in each node, minimal and maximal levels of tanks, and some similar features emerges. The other limitation includes the times of turning the pumps on and off such that the objective function is performed in both statuses. The first status of each pump's on-off status was determined three times at maximum, and the second status of each pump's on-off status was determined exactly three times. The proposed model is used in the distribution network of Van Zyl and the optimal operation instruction of derivation, and the results will be compared with those of genetic and ant colony algorithms.

The objective function in pumps optimal control problem in the distribution network is to minimize the amount of energy consumption cost during the daytime. The decision variables include the on and off statuses of available pumps in the network during different daytime times.

The objective function in this problem is defined as in Eq. (6):

$$
\begin{aligned}
\operatorname{MinCost}= & \sum_{n=1}^{N_{p}} \sum_{i=1}^{N_{T}} R_{e}(i) \cdot E_{c}(n, i) \cdot S(n, i) \\
& + \text { Penalty } 1+\text { Penalty } 2 .
\end{aligned}
$$

In the above equation, $N_{p}$ is the number of pumps, $N_{T}$ is the number of time ranges, $S(n, i)$ is the operation time of pump $n$ in time range $i, R_{e}(i)$ is the energy definition in time range $i$, and $E_{c}(n, i)$ is the energy consumption of pump $n$ in time range $(\mathrm{kW})$.

The energy consumption of a pump depends on discharge, pumping height, and pump efficiency, obtained through the following equation:

$$
E_{c}(n, i)=\frac{0.01019 \cdot Q(n, i) \cdot h(n, i)}{e(n, i)},
$$

where $Q(n, i)$ is the pumping discharge of pump $n$ in time range $(L / s) i, h(n, i)$ is the pumping height of pump $n$ in time range $i(m)$, and $e(n, i)$ is the pumping efficiency of pump $n$ in time range $i$.

In this model, the energy cost is calculated by EPANET model directly. In addition, the penalties are as follows.

- Penalty 1: The penalty that is considered for backtracking of the considered option from the allowed minimum and maximum levels of the tank. The value of this penalty is calculated through the following equation:

$$
\begin{gathered}
\text { Penalty } 1=\alpha\left\{\begin{array}{l}
\sum_{k=1}^{N t} \sum_{t=1}^{T}\left(\frac{H_{k}^{t}}{H_{\min , k}}\right)^{2} \\
\sum_{k=1}^{N t} \sum_{t=1}^{T}\left(\frac{H_{k}^{t}}{H_{\max , k}}\right)^{2}
\end{array}\right. \\
\forall\left\{\begin{array}{l}
H_{k}^{t}<H_{\min , k} \\
H_{k}^{t}>H_{\max , k}
\end{array}\right.
\end{gathered}
$$

where $k$ is the number of tanks, $N t$ is the total number of all tanks, $t$ is the number of the considered time ranges, and $T$ is the total number of time ranges where water depth values will be calculated. $H_{k}^{t}$ is the water depth value in the $k$ th tank in the $t$ th time range. $H_{\min , k}$ and $H_{\max , k}$ are minimum and maximum allowed water depths in the $k$ th tank, respectively.

- Penalty 2: This penalty is considered for the creation of depth lack. Depth lack is the saving tank depth lack at the end of the period toward its depth at the start of the period, and the value of this penalty is calculated through the following equation:

$$
\text { Penalty } 2=\beta\left\{\sum_{k=1}^{N t}\left(\frac{H_{k}^{T}}{H_{I n i, k}}-1\right)^{2}\right\},
$$

where $H_{k}^{T}$ is the water depth of the $k$ th tank at the end of the time period, and $H_{I n i, k}$ is the first water depth in the $k$ th tank.

It should be mentioned that $\alpha$ and $\beta$ are the penalty coefficients and their proper values in the studied issue obtained after trial-and-error approach are $\alpha=10^{10}$ and $\beta=10^{11}$, respectively.

On the other hand, another operation cost imposed on the system is about turning the pumps on and off. This cost will increase by an increment in the number of turning the pumps on and off. One way for performing this operation cost with respect 
to the objective function is to minimize the times of turning the pumps on and off. The calculation of the cost of turning the pumps on and off is a difficult task and cannot be done carefully; thus, it is suggested to perform the times of turning the pumps on and off as a limitation on the objective function [28]. This limitation is stated through the following equations:

$$
\begin{aligned}
& N_{S}=N_{P} \times S W, \\
& N_{S} \leq N_{P} \times S W,
\end{aligned}
$$

where $N_{S}$ is the total time of turning system pumps on and off, and $S W$ is the maximum time of turning each pump on and off. In Eq. (10), the time of turning each pump on and off is equal to $S W$; in Eq. (11), the time of turning each pump on and off is equal to $S W$ in the maximum case.

\section{Model performance and result surveying}

\subsection{Van Zyl water distribution network}

In order to study the performance of G-JPSO algorithm, Van Zyl water distribution network, presented by Van Zyl in 2004, has been solved [7]. This network includes three pumps and two tanks, as illustrated in Figure 5. The Pumps $1 \mathrm{~A}$ and $2 \mathrm{~B}$ are connected to each other in a parallel form. When each pump is activated, the Pump 3B transfers water from Tank A to Tank B. The height of Tank B is more than that of Tank A. The period of pump cycle is 24 hours, which is divided into 24 one-hour periods. At the beginning of the operation period which is considered simultaneously with the beginning of electricity cost messenger (7:00 am), 95\% of the tank volume is full of water. Therefore, the first water heights of Tank A and Tank B are 4.5 and 9.5 meters, respectively [8]. The considered problem has been solved in two statuses of $N_{s} \leq 9$ and $N_{s}=9$ with the two algorithms, JPSO and G-JPSO. In algorithm analysis, the results of 25 times of different algorithm performances were used. Each time, the performance of the objective function model was calculated 6000 times. Tables 3 and 4 show the statistical results of electricity consumption cost of distribution network pumps through 25 times of program runs with $N_{s} \leq 9$ and $N_{s}=9$ for the two algorithms, JPSO and G-JPSO. As is shown in $N_{s} \leq 9$, the results of the objective function of JPSO algorithm are better than those of Hybrid GA algorithm. In both statuses, JPSO algorithm has a better average response; the worst response, which was yielded in 25 times of the model running, was lower than that of the two others. The power of ant colony algorithm for finding the minimal response was greater than that of the two other algorithms in both statuses. By presenting the proposed mechanism, G-

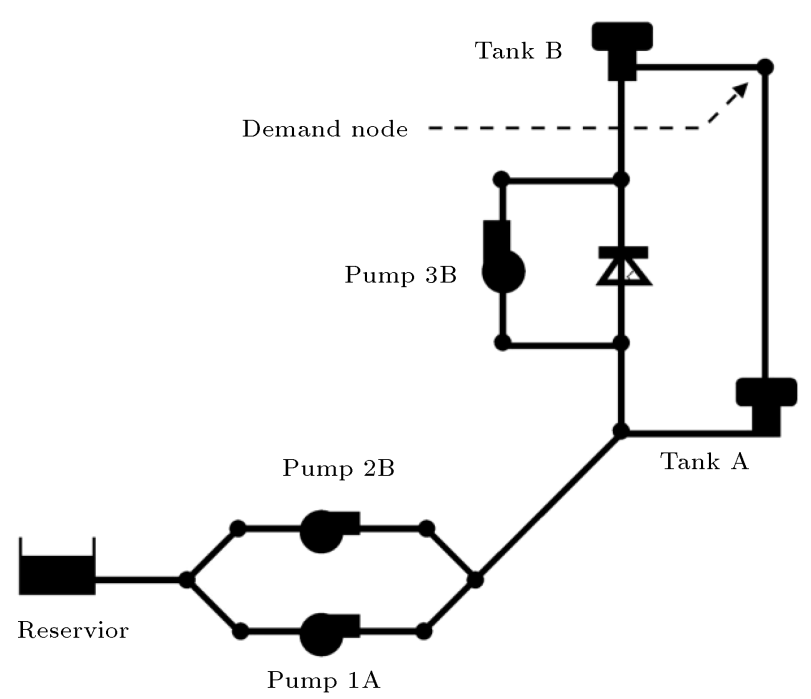

Figure 5. Van Zyl network.

Table 4. The cost of electricity and turning off of the pumps run at 25 times 6000 model and calculating the objective function $\left(N_{S}=9\right)$.

\begin{tabular}{ccccccc}
\hline & \multicolumn{6}{c}{ Algorithm } \\
\cline { 2 - 7 } & \multicolumn{3}{c}{ GJPSO } & \multicolumn{2}{c}{ JPSO } & \multicolumn{2}{c}{ ACO** $^{* *}$} \\
\cline { 2 - 7 } & $\boldsymbol{C}_{\boldsymbol{E}}$ & $\boldsymbol{N}_{\boldsymbol{S}}$ & $\boldsymbol{C}_{\boldsymbol{E}}$ & $\boldsymbol{N}_{\boldsymbol{S}}$ & $\boldsymbol{C}_{\boldsymbol{E}}$ & $\boldsymbol{N}_{\boldsymbol{S}}$ \\
\hline The best & 328.69 & 9 & 341.41 & 9 & 329.1 & $\mathbf{9}$ \\
Median & 336.36 & 9 & 347.66 & 9 & 357.9 & $\mathbf{9}$ \\
The worst & 347.58 & 9 & 355.81 & 9 & 364.7 & $\mathbf{9}$ \\
\hline
\end{tabular}

**: López et al. (2008) [9].

Table 3. The cost of electricity and turning off of the pumps run at 25 times 6000 model and calculating the objective function $\left(N_{S} \leq 9\right)$.

\begin{tabular}{lccccccccc}
\hline & \multicolumn{8}{c}{ Algorithm } \\
\cline { 2 - 10 } & \multicolumn{2}{c}{ GJPSO } & \multicolumn{2}{c}{ JPSO } & \multicolumn{2}{c}{ ACO $^{*}$} & \multicolumn{1}{c}{ Hybrid GA } \\
\cline { 2 - 10 } & $\boldsymbol{C}_{\boldsymbol{E}}$ & $\boldsymbol{N}_{\boldsymbol{S}}$ & $\boldsymbol{C}_{\boldsymbol{E}}$ & $\boldsymbol{N}_{\boldsymbol{S}}$ & $\boldsymbol{C}_{\boldsymbol{E}}$ & $\boldsymbol{N}_{\boldsymbol{S}}$ & $\boldsymbol{C}_{\boldsymbol{E}}$ & $\boldsymbol{N}_{\boldsymbol{S}}$ \\
\hline The best & 325.96 & 6 & 341.7 & 6 & 326.5 & 3 & 344.4 & $\mathbf{3}$ \\
Median & 334.91 & 6 & 344.21 & 7 & 349.2 & 4 & 348.6 & $\mathbf{4}$ \\
The worst & 349.32 & 5 & 353.11 & 9 & 357.6 & 7 & 354.8 & $\mathbf{5}$ \\
\hline
\end{tabular}

*: López-Ibáñez et al. (2008) [9]. 
Table 5. G-JPSO and JPSO algorithm parameters for solving optimal control problem pumps $\left(N_{S} \leq 9\right)$.

\begin{tabular}{ccccccc}
\hline Algorithm & $\begin{array}{c}\text { The number } \\
\text { of repetitions }\end{array}$ & $\begin{array}{c}\text { The number } \\
\text { of particles }\end{array}$ & C1 & C2 & C3 & C4 \\
\hline G-JPSO & 10 & 600 & 0.7 & 0.1 & 0.1 & 0.1 \\
JPSO & 400 & 15 & 0.55 & 0.15 & 0.15 & 0.15 \\
\hline
\end{tabular}

Table 6. G-JPSO and JPSO algorithm parameters for solving optimal control problem pumps $\left(N_{S}=9\right)$.

\begin{tabular}{ccccccc}
\hline Algorithm & $\begin{array}{c}\text { The number } \\
\text { of repetitions }\end{array}$ & $\begin{array}{c}\text { The number } \\
\text { of particles }\end{array}$ & C1 & C2 & C3 & C4 \\
\hline G-JPSO & 12 & 500 & 0.7 & 0.1 & 0.1 & $\mathbf{0 . 1}$ \\
JPSO & 400 & 15 & 0.55 & 0.15 & 0.15 & $\mathbf{0 . 1 5}$ \\
\hline
\end{tabular}

Table 7. The cost of electricity varying with the number of repetitions and the number of particles $\left(N_{S} \leq 9\right)$.

\begin{tabular}{cccc}
\hline Algorithm & $\begin{array}{c}\text { The number of } \\
\text { repetitions }\end{array}$ & $\begin{array}{c}\text { The number of } \\
\text { particles }\end{array}$ & $\begin{array}{c}\text { Minimum cost } \\
\text { (dollar) }\end{array}$ \\
\hline \multirow{3}{*}{ G-JPSO } & 600 & 10 & $\mathbf{3 3 0 . 3}$ \\
& 500 & 14 & $\mathbf{3 2 8 . 5 9}$ \\
& 400 & 15 & $\mathbf{3 3 3 . 8 1}$ \\
& 200 & 30 & $\mathbf{3 3 8 . 3 2}$ \\
12 & 500 & $\mathbf{3 3 0 . 2 6}$ \\
& 600 & 600 & $\mathbf{3 2 5 . 9 6}$ \\
JPSO & 500 & 10 & $\mathbf{3 4 4 . 4}$ \\
& 400 & 14 & $\mathbf{3 4 6 . 1}$ \\
& 10 & 15 & $\mathbf{3 4 1 . 7}$ \\
& 600 & 30 & $\mathbf{3 4 2 . 7}$ \\
& 12 & 500 & $\mathbf{3 4 3 . 3}$ \\
& & 10 & $\mathbf{3 4 2 . 5}$ \\
\hline
\end{tabular}

Table 8. The cost of electricity varying with the number of repetitions and the number of particles $\left(N_{S}=9\right)$.

\begin{tabular}{cccc}
\hline Algorithm & $\begin{array}{c}\text { The number of } \\
\text { repetitions }\end{array}$ & $\begin{array}{c}\text { The number of } \\
\text { particles }\end{array}$ & $\begin{array}{c}\text { Minimum cost } \\
\text { (dollar) }\end{array}$ \\
\hline \multirow{3}{*}{ G-JPSO } & 600 & 10 & $\mathbf{3 3 4 . 6 3}$ \\
& 500 & 14 & $\mathbf{3 3 7 . 3 8}$ \\
& 400 & 15 & $\mathbf{3 3 0 . 9 7}$ \\
& 200 & 30 & $\mathbf{3 3 5 . 5 7}$ \\
12 & 500 & $\mathbf{3 3 1}$ \\
& 600 & 600 & $\mathbf{3 2 8 . 6 9}$ \\
JPSO & 500 & 10 & $\mathbf{3 4 8 . 3 8}$ \\
& 400 & 14 & $\mathbf{3 4 5 . 6 3}$ \\
& 10 & 15 & $\mathbf{3 4 1 . 4 1}$ \\
& 600 & 30 & $\mathbf{3 4 7 . 1 7}$ \\
& 12 & 500 & $\mathbf{3 4 5 . 6 2}$ \\
& & 10 & $\mathbf{3 4 3 . 5 7}$ \\
\hline
\end{tabular}

JPSO algorithm reaches a better response than that of ant colony algorithm. By applying the proposed algorithm and making a graph for solving the problem, the searching space has decreased and the possibility to reach an optimal response has increased (the searching space decreased from the status $2^{24}=16,777,216$ to the status 290,998 for $N_{s} \leq 3$ for each pump). Tables 5 and 6 list the parameters of JPSO and GJPSO algorithms for solving the problem of pumps optimal control with $N_{s} \leq 9$ and $N_{s}=9$. Tables 7 and 8 show the statistical results of electricity consumption cost of distribution network pumps in 25 times of 


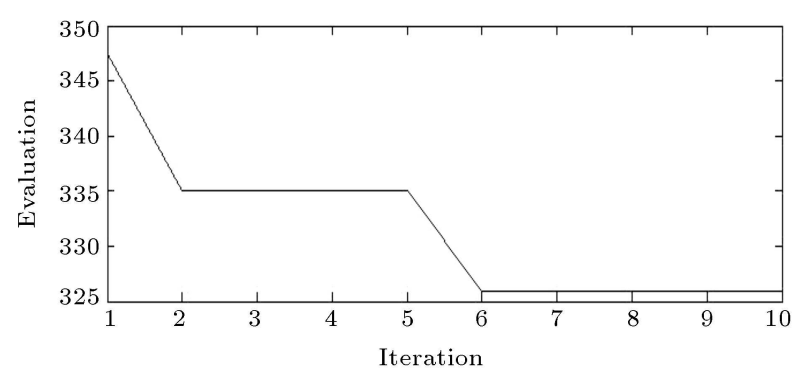

Figure 6. Convergence of optimal solution and optimal control problem in the algorithm pumps G-JPSO $\left(N_{S} \leq 9\right)$.

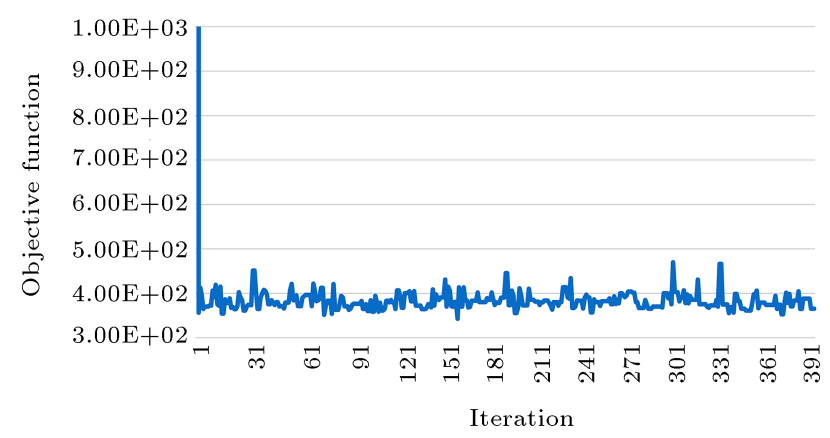

Figure 7. Convergence of optimal solution and optimal control problem in the algorithm pumps JPSO $\left(N_{S} \leq 9\right)$.

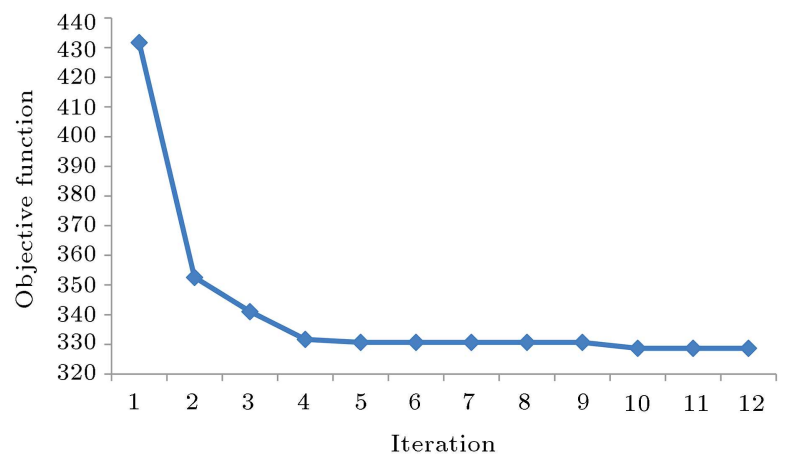

Figure 8. Convergence of optimal solution and optimal control problem in the algorithm pumps G-JPSO $\left(N_{S}=9\right)$.

program running with $N_{s} \leq 9$ and $N_{s}=9$ for the two algorithms, JPSO and G-JPSO. Figures 6-7 and Figures 8-9 respectively show the optimal response convergence of the pumps optimal control problem in JPSO and G-JPSO algorithms with $N_{s} \leq 9$ and $N_{s}=$ 9. Figures 10 and 11 depict the operation instruction from available pumps in the network during 24 hours of the operation period with $N_{s} \leq 9$ and $N_{s}=9$ resulting from G-JPSO and JPSO algorithms.

\subsection{Richmond real network}

In order to examine the efficiency of the presented model, the proposed algorithm is also applied to the Richmond water distribution system, which is a real system located in England [8]. The calibrated network

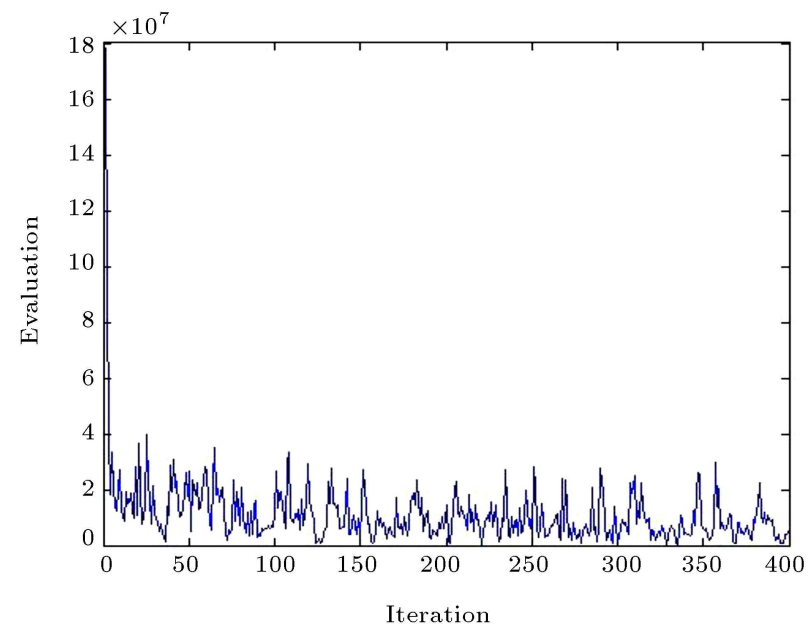

Figure 9. Convergence of optimal solution and optimal control problem in the algorithm pumps JPSO $\left(N_{S}=9\right)$.

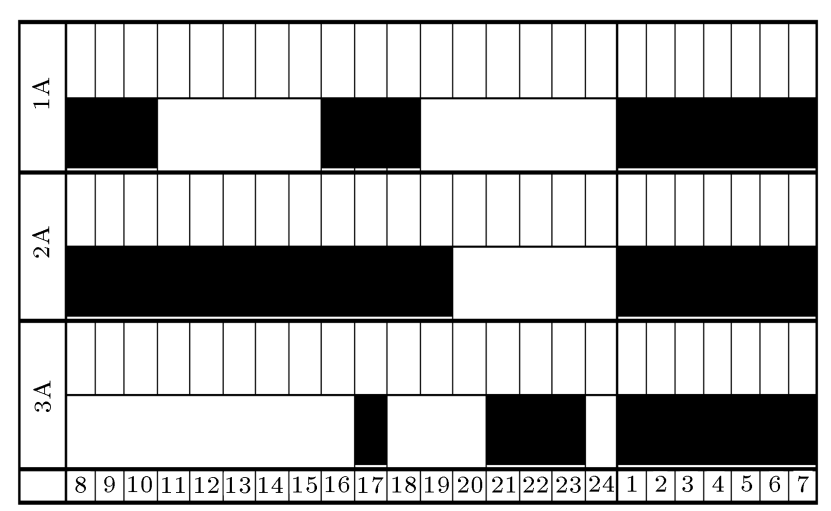

Figure 10. Optimal on and off turning of the pump during a 24-hour period $\left(N_{S} \leq 9\right)$.

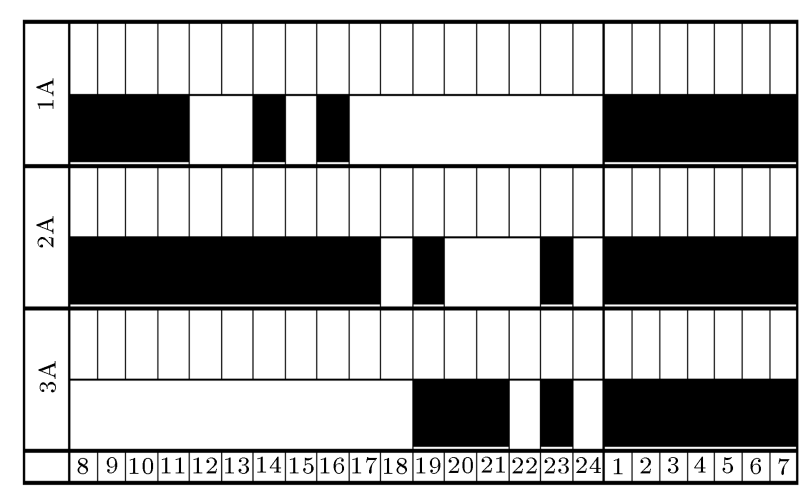

Figure 11. Optimal on and off turning of the pump during a 24-hour period $\left(N_{S}=9\right)$.

has 7 pumps, 6 tanks, 1 reservoir, 948 links, and 836 nodes (Figure 12). This network was studied for the first time by Atkinson et al. (2000) [29]. All the tanks of the network should be filled up to $95 \%$ at the beginning of the period of electricity peak. Table 9 shows the statistical results of electricity consumption cost of distribution network pumps in 25 times of program 
Table 9. Cost of electricity in Richmond.

\begin{tabular}{|c|c|c|c|c|c|c|c|c|}
\hline & \multicolumn{8}{|c|}{ Algorithm } \\
\hline & \multirow{2}{*}{\multicolumn{2}{|c|}{$\begin{array}{c}\text { G-JPSO } \\
(8000 \text { evaluations })(800\end{array}$}} & \multirow{2}{*}{$\begin{array}{l}\text { JPSO } \\
0 \text { evaluation }\end{array}$} & \multicolumn{2}{|c|}{ ACO $^{*}$} & \multicolumn{2}{|c|}{$\mathrm{ACO}^{*}$} & $\mathbf{G A}^{*}$ \\
\hline & & & & $(8000$ ev & aluations) & $(50000$ ev & valuations) & (100000 evaluations) \\
\hline & $N_{S} \leq \mathbf{2 1}$ & $N_{S}=\mathbf{2 1}$ & $N_{S} \leq \mathbf{2 1}$ & $N_{S} \leq \mathbf{2 1}$ & $N_{S}=\mathbf{2 1}$ & $N_{S} \leq \mathbf{2 1}$ & $N_{S}=\mathbf{2 1}$ & $N_{S} \leq \mathbf{9}$ \\
\hline The best & 24805.4 & 32631 & 37314.7 & 33683.3 & 34319.9 & 32728.5 & 32581.4 & 37266.5 \\
\hline Median & 30116.1 & 35576.5 & 37697.8 & 36069.7 & 36699.7 & 33747.5 & 33634.1 & 41099 \\
\hline The Worst & 33689.5 & 36500 & 42782.3 & 38017.3 & 39052.4 & 35745.5 & 34464.8 & 43508 \\
\hline
\end{tabular}

*: López-Ibáñez et al. 2008 [9].

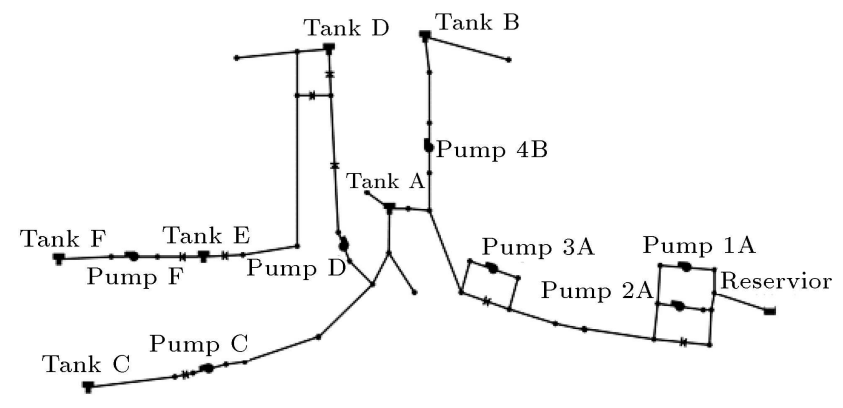

Figure 12. Richmond network.

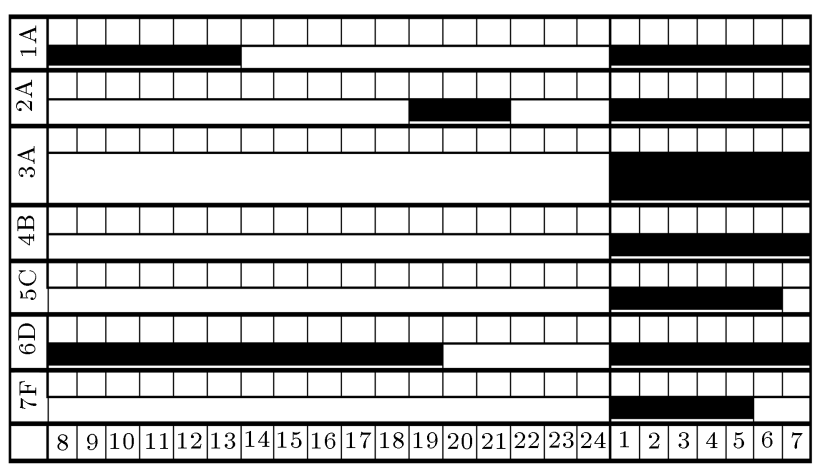

Figure 13. Optimal on and off turning of the pump during a 24-hour period $\left(N_{S} \leq 21\right)$.

running with $N_{s} \leq 21$ and $N_{s}=21$ for the two algorithms, JPSO and G-JPSO. The comparison of the obtained results and those of the ant colony and genetic algorithm shows the superiority of the proposed G-JPSO algorithm.

Figures 13 and 14 show the operation instructions from available pumps in the network during 24 hours of operation period with $N_{s} \leq 21$ and $N_{s}=21$ resulting from G-JPSO and JPSO algorithms.

\section{Conclusion}

In this research, by applying some changes to the jumping nature in JPSO algorithm, the ability to solve the discrete graphic issues was considered. This algorithm is named G-JPSO. At first, the proposed algorithm was used for solving the mathematical problem

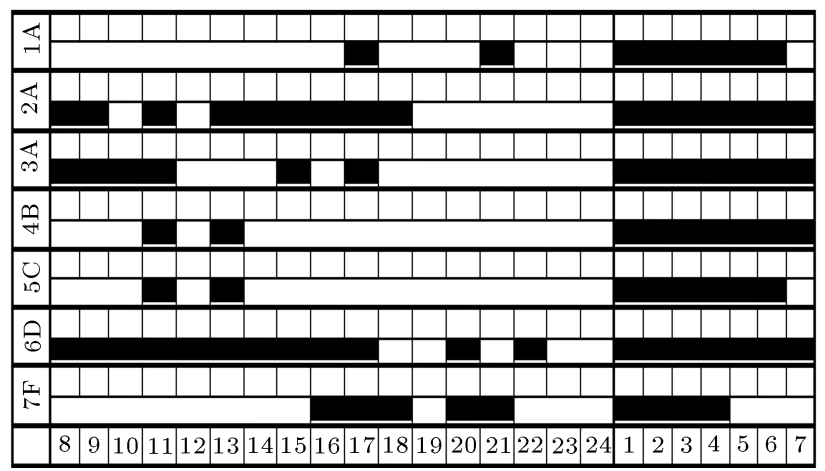

Figure 14. Optimal on and off turning of the pump during a 24-hour period $\left(N_{S}=21\right)$.

of minimizing Fletcher-Powell complex function, and the observed responses were much better than those of ant colony algorithm. To determine the pumps optimal operation program, a simulation-optimization model was proposed based on G-JPSO and JPSO algorithms. Operation program includes determining on and off statuses of each pump during the daytime in the time range of 1 hour. Thus, in addition to a decrease in the electricity consumption cost, the need for low-consuming nodes, and problem limitations including minimal required pressure in each node, the minimal and maximal levels of tanks were investigated. The other limitation includes the times of turning the pumps on and off where the objective function is performed in both statuses. The proposed model was used in the distribution network of Van Zyl and optimal operation instruction of derivation, and the related results were compared with those of genetic and ant colony algorithms. The results show that the response of objective function of JPSO algorithm was better than that of hybrid GA algorithm; however, the power of G-JPSO algorithm for finding the minimal response was more than that of the JPSO, Hybrid GA, and ant colony algorithms in both statuses. However, generally, the response of G-JPSO algorithm yielded in 25 times of the model run was less worse than those of the three others. In addition, the proposed model was evaluated for Richmond real network, and the significant superiority of the proposed algorithm 
over genetic and ant colony algorithms was considered. This excellence results from solving these problems by making a graph that leads to a decrease in the searching space and an increase in the possibility to reach an optimal response. In this research, by creating this ability in JPSO algorithm, the searching space decreased, and the power of the algorithm for finding an optimal response increased. The required average times for program run by a laptop equipped with a Core i5-2430M $2.4 \mathrm{GHz}$ for Van Zyl network with 6000 iterations by G-JPSO and JPSO are 318 and 408 seconds and are 7816 and 8317 seconds for Richmond network. The computational effort required by the ACO approach was measured when run on a Pentium $4(3.20 \mathrm{GHz})$ with $1,024 \mathrm{kB}$ of cache size and running under Red Hat GNU/Linux with kernel version 2.4.20. The mean computation time required for 6,000 evaluations of the van Zyl network was $417.6 \mathrm{~s}$, while it was $8,862 \mathrm{~s}$ in the case of 8,000 evaluations of the Richmond network [9]. It is recommended that the proposed algorithm be used for solving the problems on the real scale, and other problems are of graphic nature.

\section{References}

1. Yeniay, O. "A comparative study on optimization methods for the constrained nonlinear programming problems", Mathematical Problems in Engineering, 2, pp. $165-173$ (2005).

2. Lee, K.S., Geem, Z.W., Lee, S.H., and Bae, K.W. "The harmony search heuristic algorithm for discrete structural optimization", Eng. Optim., 37, pp. 663$684(2005)$.

3. Afshar, H. and Rajabpour, R. "Application of local and global particle swarm optimization algorithms to optimal design and operation of irrigation pumping systems", Irrig. and Drain., 58(3), pp. 321-331 (2009).

4. Mackle, G., Savic, D.A., and Walters, G.A. "Application of genetic algorithms to pump scheduling for water supply", GALESIA, 95. London: Institute of Electrical Engineers Conference Publication, 4(4), pp. 400-405 (1995).

5. Rodin, S.I. and Moradi-Jalal, M. "Use of genetic algorithm in optimization of irrigation pumping stations", WAPIRRA program. [Online]. <http://stullia.t-k. $\mathrm{ru} /$ waterpump/waterpump.htm $>$. (June 10, 2002).

6. Moradi-Jalal, M., Marino, M.A., and Afshar, A. "Optimal design and operation of irrigation pumping station", J. Irrig. Drain. Eng., 129(3), pp. 149-154 (2003).

7. Rajabpour, R. and Afshar, M.H. "Optimized operation of serial pump stations using the PSO algorithm", Journal of Water \& Wastewater, 66, pp. 56-66 (2008).

8. Van Zyl, J.E., Savic, D.A., and Walters, G.A. "Operational optimization of water distribution systems using a hybrid genetic algorithm", J. Water Resour. Plann. Manage., 130(2), pp. 160-170 (2004).

9. López-Ibáñez, M., Prasad, T.D., and Paechter, B. "Ant colony optimization for optimal control of pumps in water distribution networks", J. Water Resour. Plann. Manage., 134(4), pp. 337-346 (2008).

10. Bozorg Haddad, O. and Marino, M.A ."Dynamic penalty function as a strategy in solving water resources combinatorial optimization problems with honey-bee mating optimization (HBMO) algorithm", Journal of Hydroinformatics, 9(3), pp. 233-250 (2007).

11. Sanda-Carmen, G., Radu, P., and Andrei-Mugur, G. "Pumping stations scheduling for a water supply system with multiple tanks", U.P.B. Sci. Bull., Series D, 72(3), pp. 129-140 (2010).

12. Rasoulzadeh Gharibdosti, S. and Bozorghadad, O. "Development and application of NLP-GA hybrid algorithm to optimize the design and operation of pumping stations", Iranian Journal of Soil and Water Research, 43(2), pp. 129-137 (2012).

13. Hashemi, S.S., Tabesh, M., and Ataee Kia, B. "Antcolony optimization of energy cost in water distribution systems using variable speed pumps", In Proceedings of 4th ASCE-EWRI International Perspective on Water Resources and The Environment, 4-6 January, National University of Singapore, Singapore (2011).

14. Hashemi, S.S., Tabesh, M., and Ataee Kia, B. "Scheduling and operating costs in water distribution networks", Water Management, 166(8), pp. 432-442 (2012).

15. Mehzad, N., Tabesh, M., and Hashemi, S.S. "Reliability of water distribution networks due to pumps failure: comparison of VSP and SSP application", Drinking Water Engineering and Science, 5, pp. 351-373 (2012).

16. Hashemi, S.S., Tabesh, M., and Ataee Kia, B. "Antcolony optimization of pumping schedule to minimize the energy cost using variable-speed pumps in water distribution networks", Urban Water Journal, 11(5), pp. 335-347 (2014).

17. Abdelmeguid, H. and Ulanicki, B. "Feedback rules for operation of pumps in a water supply system considering electricity tariffs", In Water Distribution Systems Analysis, pp. 1188-1205 (2010).

18. Skworcow, P., Ulanicki, B., AbdelMeguid, H., and Paluszczyszyn, D. "Model predictive control for energy and leakage management in water distribution systems", In UKACC International Conference on Control, Coventry, UK (2010).

19. Fiorelli, D., Schutz, G., Metla, N., and Meyers, J. "Application of an optimal predictive controller for a small water distribution network in Luxembourg", Journal of Hydroinformatics, 15(3), p. 625 (2013). DOI: $10.2166 /$ hydro.2012.117 
20. Paluszczyszyn, D., Skworcow, P., and Ulanicki, B. "Online simplification of water distribution network models for optimal scheduling", Journal of Hydroinformatics, 15(3), pp. 652-665 (2013). DOI: $10.2166 /$ hydro. 2012.029

21. Kennedy, J. and Eberhart, R. "Adiscrete binary version of the particle swarm algorithm", In IEEE Conference on Systems, Man, and Cybernerics, 5, pp. 4104-4108 (1997).

22. Yang, S., Wang, M., and Jiao, L. "A quantum particle swarm optimization", In Proceedings of CEC2004, the Congress on Evolutionary Computing, 1, pp. 320-324 (2004).

23. Al-Kazemi, B. and Mohan, C.K. "Multi-phase discrete particle swarm optimization", In Fourth International Workshop on Frontiers in Evolutionary Algorithms, Kinsale, Ireland (2002).

24. Moreno-Perez, J.A., Castro-Gutierrez, J.P, MartinezGarcia F.J., Melian, B., Moreno-Vega, J.M., and Ramos, J. "Discrete particle swarm optimization for the p-median problem", In Procceedings of the 7th Metaheuristics International Conference, Montreal, Canada (2007).

25. Sami Kashkoli, B. and Monem, J. "Development and application of pressure irrigation systems using integrated optimization model JPSO / LIDM", 8th Iranian Hydraulic Conference, Azar, Tehran (2009).

26. Gen, M. and Cheng, R.W., Genetic Algorithm and Engineering Design, John Wiley and Sons, Inc (1997).

27. Jalali, M.R. and Afshar, A. "Optimum design and operation of hydrosystem by ant colony optimization algorithm; A New Metaheuristic Approach", Ph.D. Dissertation of Water Engineering, Iran University of Science and Technology (2005).

28. Lansey, K.E. and Awumah, K. "Optimal pump operations considering pump switches", J. Water Resour. Plann. Manage., 120(1), pp. 17-35 (1994).
29. Atkinson, R., van Zyl, J.E., Walters, G.A., and Savic, D.A. "Genetic algorithm optimization of levelcontrolled pumping station operation", Proc., Water Network Modelling for Optimal Design and Management, Centre for Water Systems, Exeter, U.K., pp. 79-90 (2000).

\section{Biographies}

Rasoul Rajabpour received his BS degree in Agricultural Engineering from Shiraz University, Shiraz, Iran in 2004 and MSc degree in Civil Engineering from University of Science and Technology, Tehran, Iran in 2007. His research interests include water management, optimization, heuristic algorithms, and water distribution systems. Now, he is a lecturer at Islamic Azad University and a prospective $\mathrm{PhD}$ student.

Nasser Talebbeydokhti, Professor of Civil and Environmental Engineering from School of Engineering of Shiraz University, is a member of Academy of Sciences and was recognized as a distinguished Professor in 2017 by Ministry of Higher Education. He is currently the Editor-in-Chief of Iranian Journal of Science and Technology Transaction of Civil Engineering. He also won the prize for the best engineering book in 1990 .

Gholamreza Rakhshandehroo was born on December 13th, 1959. He obtained his bachelor degree in Civil Engineering from Shiraz University, Shiraz, Iran, July 1985. He worked as an inspector engineer and, then, a chief engineer at Shiraz Municipality from 1985 to 1989. He then moved to USA and did his masters and PhD in Civil and Environmental Engineering at Michigan State University. He returned to Shiraz University and taught there since 1997. He is currently a Full Professor in Civil and Environmental Engineering and serves as the Vice Chancellor in students' and cultural affairs for the university. 